\title{
Infecciones vaginales y complicaciones durante el embarazo en usuarias del Centro de Salud Universitario de Motupe - Loja
}

\author{
Vaginal infections and complications during pregnancy in users of Motupe University \\ Health Center - Loja
}

\author{
María de los Ángeles Sánchez Tapia ${ }^{1, *}$ y Viviana Yomary González Armijos ${ }^{1}$ \\ ${ }^{1}$ Carrera de Medicina Humana, Universidad Nacional de Loja, Loja, Ecuador \\ *Autor para correspondencia: maria.a.sanchez@unl.edu.ec
}

Fecha de recepción del manuscrito: 15/07/2021 Fecha de aceptación del manuscrito: 12/12/2021 Fecha de publicación: 24/12/2021

\begin{abstract}
Resumen-Las infecciones vaginales son corresponsables de un importante porcentaje de morbilidad materna y perinatal, presentando complicaciones como amenaza de aborto, ruptura de membranas, parto pretérmino y enfermedad pélvica inflamatoria, permitiendo con este trabajo investigativo la elaboración de un perfil clínico-epidemiológico, favoreciendo al personal médico y usuarias gestantes, contribuyendo a la detección oportuna desde el primer nivel de atención, disminuyendo sus complicaciones y mejorando su calidad de vida; cuyos objetivos fueron: Determinar las infecciones vaginales y complicaciones durante el embarazo, conocer las infecciones vaginales más frecuentes, identificar las complicaciones y establecer la relación entre estas ; siendo un estudio descriptivo, con enfoque cualitativo y cuantitativo desarrollado en el Centro de Salud Universitario de Motupe en el período Octubre 2020 - Marzo 2021, cuyo universo constituyó 127 usuarias y una muestra de 52 gestantes que cumplieron con los criterios de inclusión plasmándose en una hoja de recolección de datos realizada por la autora; determinando que la Vaginosis Bacteriana se presenta en el 15\% entre los 26 - 30 años, $13 \% 21$ - 35 años, $8 \% 36$ 40 años y $6 \% 16$ - 20 y 31 - 35 años, tricomoniasis $6 \% 21$ - 35 años, el 2\% 26 - 35 años, candidiasis $13 \%$ en 26 - 30 años, $9 \%$ 21 - 35 años, el 8\% 16-20 años, 6\% 31 - 40 años, como complicación el $4 \%$, amenaza de aborto y el $96 \%$ sin complicaciones. Las infecciones vaginales, vaginosis bacteriana y candidiasis fueron las que presentaron complicaciones de amenaza de aborto en un 4,38\%, evidenciándose que las pacientes que presentan infecciones vaginales no muestran complicaciones en alto porcentaje.
\end{abstract}

Palabras clave-Complicaciones, Embarazo, Infección vaginal.

\begin{abstract}
Vaginal infections are co-responsible for a significant percentage of maternal and perinatal morbidity due to complications such as threatened abortion, ruptured membranes, preterm delivery and pelvic inflammatory disease. This research paper resulted in the creation of clinical-epidemiological profiles, favoring medical staff and pregnant users. They contributed to the timely detection from the very first level of care, thus reducing complications and improving their quality of life. The objectives of this study were: to determine vaginal infections and complications during pregnancy; to recognize the most frequent vaginal infections; to identify complications and to establish the relationship between them. This report is a descriptive, ambispective, correlational and cross-sectional study, employing both a qualitative and quantitative approach. This was carried out at the Motupe University Health Center during the period October 2020 - March 2021. The sample consisted of 127 users and a sample of 52 pregnant women who met the inclusion criteria. Details were inputted into a data collection sheet created by the author. Results showed that Bacterial Vaginosis occurred in $15 \%$ of women between 26-30 years of age, $13 \%$ in women between 21-35 years of age, $8 \%$ in women between 36-40 years of age and 6\% in women between 16-20 years of age and women aged between 31-35 years old. Tricomoniasis occurred in 6\% of women aged 21-35 years of age, $2 \%$ in those aged 26-35 and Candidiasis occurred in $13 \%$ of women aged 26-30 years, $9 \%$ in women aged 21-35, $8 \%$ in those aged $16-20$ and $6 \%$ in those aged $31-40$. With respect to complications the study showed $4 \%$ suffered from the threat of abortion and $96 \%$ did not manifest any complications as a result of these infections. From bacterial vaginosis and candidiasis infections $4.38 \%$ presented complications with respect to the threat of abortion thus demonstrating that patients with vaginal infections do not display complications in high percentages.
\end{abstract}

Keywords-Complications, Pregnancy, Vaginal infection.

\section{INTRODUCCIÓN}

L as infecciones vaginales constituyen una de las razones más frecuentes de consultas prenatales y son corres- ponsables de un importante porcentaje de morbilidad materna y morbilidad y mortalidad perinatal, sobre todo en lugares de escasos recursos. Las mujeres embarazadas desarrollan de manera fácil infecciones vaginales debido a cambios funcio- 
nales y hormonales (MSP, 2014).

En la práctica médica las infecciones vaginales constituyen un problema de salud frecuente, con una incidencia mundial del $7 \%$ al $20 \%$ de las mujeres por año. La vaginitis se encuentra dentro de los diez motivos de consulta más comunes en medicina general y constituyen el $15 \%$ - $20 \%$ de consultas en la práctica ginecológica. Aproximadamente el $75 \%$ de todas las mujeres tendrán una infección vaginal fúngica al menos una vez en la vida, de las cuales, el $40 \%$ - $50 \%$ son recurrentes (Milhet, et al., 2016).

Según la OMS (Organización Mundial de la Salud), la vaginosis bacteriana es relativamente común. La prevalencia informada en embarazadas oscila entre el $14 \%$ y el $21 \%$ en países occidentales. En Asia, la prevalencia informada de vaginosis bacteriana durante el embarazo es del 13,6\% en mujeres japonesas, del $15,9 \%$ en las tailandesas y del $18 \%$ en las de Indonesia.

En Ecuador, en el 2016 de acuerdo con el reporte anual realizado por el Instituto Nacional de Estadísticas y Censos la infección de las vías genitourinarias en el embarazo figura dentro de las diez principales causas de morbilidad femenina. (INEC, 2016). En un estudio realizado en el servicio de Gineco-Obstetricia del Hospital Isidro Ayora de la Ciudad de Loja la prevalencia de Vulvovaginitis corresponde al 6,17\%, de las cuales el $62,66 \%$ correspondieron a Candidiasis vulvovaginal, el $27,92 \%$ a Vaginosis bacteriana y el $9,42 \%$ a Tricomoniasis vaginal (Jumbo, 2017).

Por consiguiente, las infecciones vaginales y complicaciones, establecen un problema de salud para la gestante, para el personal de salud y para las instituciones sanitarias, en este sentido se planteó la siguiente pregunta de investigación: ¿Cuáles son las infecciones vaginales y complicaciones más frecuentes en el embarazo en usuarias del Centro de Salud Universitario de Motupe?

La importancia de este trabajo de investigación radica en el hecho de que existe poca información estadística sobre esta patología a nivel local, por lo tanto los resultados obtenidos de este trabajo servirán de apoyo al personal médico así como a sus usuarias en estado de gestación que acuden al Centro de Salud Universitario de Motupe, contribuyendo así a la detección oportuna desde el primer nivel de atención, además de disminuir la frecuencia de complicaciones lo que ayudará a mejorar su calidad de vida.

La factibilidad del presente trabajo investigativo se sustentó por la autorización de las autoridades del Centro de Salud Universitario de Motupe para la recolección de la información necesaria que aseguró el cumplimiento de los objetivos planteados como: Determinar las infecciones vaginales y complicaciones durante el embarazo y cuyos objetivos específicos son: Conocer las infecciones vaginales más frecuentes durante el embarazo de acuerdo a su edad, identificar las complicaciones y relacionar tanto infecciones como complicaciones durante el embarazo en usuarias con infecciones vaginales del Centro de Salud Universitario de Motupe-Loja.

Este estudio se vinculó además a las áreas de investigación de la Universidad Nacional de Loja de la Facultad de Salud Humana, línea de investigación $\mathrm{N}^{\circ} 1$ que trata sobre la Salud Enfermedad materno-infantil en Loja y la Zona 7 del país.

\section{MATERIALES Y MÉTODOS}

La presente investigación se realizó en el del Centro de Salud Universitario de Motupe que se encuentra ubicado en el Barrio Motupe Bajo que pertenece a la Parroquia San Juan del Valle en el período octubre 2020 marzo 2021. Con un enfoque cualitativo y cuantitativo, utilizando un tipo de diseño descriptivo. La unidad de estudio y universo fueron 127 usuarias en estado gestante que acudieron a consulta externa de Gineco-Obstetricia del Centro de Salud Universitario de Motupe de la Ciudad de Loja, con diagnóstico de Infecciones Vaginales mientras que la muestra figuró 52 pacientes embarazadas que cumplieron con los criterios de inclusión que fueron los siguientes: pacientes con diagnóstico confirmado por laboratorio de algún tipo de infección vaginal, pacientes que hayan iniciado tratamiento para infecciones vaginales y pacientes gestantes de toda edad.

La técnica que se utilizó fue una encuesta elaborada por las autoras, además de una revisión y análisis de historias clínicas donde se recolectaron datos que fueron plasmados en la hoja de recolección para el desarrollo de la presente investigación con previa autorización por parte de las autoridades del Centro de Salud Universitario. Luego de la recolección de datos estos fueron analizados y tabulados con sus respectivas tablas estadísticas mediante la ayuda del programa Microsoft EXCEL 2013, de esta manera se planteó las respectivas conclusiones y recomendaciones.

\section{RESULTADOS}

La vaginosis bacteriana se presentó en el $15 \%(n=8)$ entre los 26-30 años y en un 13\% $(n=7)$ entre los 21-25 años. En tricomoniasis 6\% $(n=3)$ 21-35 años, el 2\% $(n=1)$ 26-35 años. Candidiasis $13 \%(\mathrm{n}=7)$ en $26-30$ años y en un $9 \%$ $(n=5)$ entre 21-35 años (Tabla 1).

Tabla 1: : Infecciones vaginales más frecuentes durante el embarazo de acuerdo a su edad en usuarias del Centro de Salud Universitario de Motupe -Loja

\begin{tabular}{cccc}
\hline $\begin{array}{c}\text { Infecciones } \\
\text { Vaginales }\end{array}$ & Edad & $\begin{array}{c}\text { Nro de } \\
\text { Casos }\end{array}$ & \% \\
\hline \multirow{4}{*}{$\begin{array}{c}\text { Vaginosis } \\
\text { Bacteriana }\end{array}$} & nov-15 & 0 & 0 \\
\cline { 2 - 4 } & $16-20$ & 3 & 6 \\
\cline { 2 - 4 } & $21-25$ & 7 & 13 \\
\cline { 2 - 4 } & $26-30$ & 8 & 15 \\
\cline { 2 - 4 } & $31-35$ & 3 & 6 \\
\hline \multirow{5}{*}{ Tricomoniasis } & nov-15 & 4 & 8 \\
\cline { 2 - 4 } & $16-20$ & 0 & 0 \\
\cline { 2 - 4 } & $21-35$ & 3 & 6 \\
\cline { 2 - 4 } & $26-30$ & 1 & 2 \\
\cline { 2 - 4 } & $31-35$ & 1 & 2 \\
\cline { 2 - 4 } & $36-40$ & 0 & 0 \\
\cline { 2 - 4 } Candidiasis & nov-15 & 0 & 0 \\
\cline { 2 - 4 } & $16-20$ & 4 & 8 \\
\cline { 2 - 4 } & $21-35$ & 5 & 9 \\
\cline { 2 - 4 } & $26-30$ & 7 & 13 \\
\cline { 2 - 4 } Total & $31-35$ & 3 & 6 \\
\cline { 2 - 4 } & $36-40$ & 3 & 6 \\
\cline { 2 - 4 } & & 52 & 100 \\
\hline
\end{tabular}


En la Tabla 2 se presentan los principales resultados de las complicaciones durante el embarazo en usuarias con infecciones vaginales del Centro de Salud Universitario de Motupe - Loja, del $100 \%(n=52)$ de las embarazadas que presentaron infecciones vaginales, el $4 \%(n=2)$ amenaza de aborto y el $96 \%(n=50)$ sin complicaciones.

Tabla 2: Complicaciones durante el embarazo en usuarias con infecciones vaginales del Centro de Salud Universitario de Motupe - Loja.

\begin{tabular}{lccccc}
\hline & $\begin{array}{c}\text { Nro } \\
\text { de } \\
\text { Casos }\end{array}$ & $\%$ & $\begin{array}{c}\text { Complica- } \\
\text { ciones }\end{array}$ & $\begin{array}{c}\text { Nro. } \\
\text { de } \\
\text { Casos }\end{array}$ & $\%$ \\
\hline & & $\begin{array}{c}\text { Amenaza } \\
\text { de aborto }\end{array}$ & 2 & 4 \\
\hline $\begin{array}{l}\text { Infecciones } \\
\text { Vaginales }\end{array}$ & 52 & 100 & $\begin{array}{c}\text { Sin } \\
\text { complica- } \\
\text { ciones }\end{array}$ & 50 & 96 \\
\hline \multicolumn{7}{c}{ Total } & & 52 & 100 \\
\hline
\end{tabular}

En lo referente a la relación entre infecciones vaginales y complicaciones durante el embarazo, del $100 \% \quad(n=52)$ el $48,07 \% \quad(n=25)$ presentaron vaginosis bacteriana y el $42,30 \%$ candidiasis, presentando complicaciones amenaza de aborto el $2,08 \%(n=1)$ y $2,3 \%(n=1)$ respectivamente $(\mathrm{Ta}-$ bla 3).

Tabla 3: Relación entre infecciones vaginales y complicaciones durante el embarazo en usuarias del Centro de Salud Universitario de Motupe -Loja.

\begin{tabular}{|c|c|c|c|c|c|}
\hline $\begin{array}{c}\text { Infecciones } \\
\text { Vaginales }\end{array}$ & $\begin{array}{l}\text { Nro de } \\
\text { Casos }\end{array}$ & $\%$ & $\begin{array}{l}\text { Complica- } \\
\text { ciones }\end{array}$ & $\begin{array}{c}\text { Nro. } \\
\text { de } \\
\text { casos }\end{array}$ & $\%$ \\
\hline $\begin{array}{c}\text { Vaginosis } \\
\text { Bacteriana }\end{array}$ & 25 & 48,07 & $\begin{array}{l}\text { Amenaza } \\
\text { de Aborto }\end{array}$ & 1 & 2,1 \\
\hline Candidiasis & 22 & 42,3 & $\begin{array}{l}\text { Amenaza } \\
\text { de Aborto }\end{array}$ & 1 & 2,3 \\
\hline Tricomoniasis & 5 & 9.61 & & & \\
\hline Total & 52 & 100 & & 2 & 4,4 \\
\hline
\end{tabular}

\section{DISCUSIÓN}

En el orden de lo mencionado, el presente trabajo pudo constatar que la vaginosis bacteriana en usuarias del Centro de Salud Universitario de Motupe en Loja se presentó en el $15 \%$ entre los 26 - 30 años, de manera muy similar a un estudio realizado en pacientes embarazadas en la Clínica de los Condes en Chile en donde el $16 \%$ se presentó en una edad promedio de 26,5 años (Velázquez, 2016). Santana (2018) encontró un porcentaje más elevado en su investigación realizada en mujeres embarazadas atendidas en el servicio de ginecología del Hospital General del Puyo donde el 35\% se encontraron entre 20 y 30 años de edad, así mismo el $13 \%$ de pacientes con vaginosis bacteriana se encontró en la edad de 21 - 25 años un porcentaje mayor en los resultados realizados en un estudio transversal, descriptivo en pacientes gestantes de una Clínica de alta complejidad de la Ciudad de Medellín y en Colombia el 24,1\% en una mediana de edad de 23 años (Rojas, 2016), se pudo evidenciar que en otras investigaciones como en la realizada en Perú que tiene como tema "Influencia de las características maternas sobre las infeccio- nes vaginales en el Embarazo en Pacientes Atendidas en el Centro de Salud Santa Adriana” el $35 \%$ presentó vaginosis bacteriana en mayores de 35 años (Tapia, 2020).

Las infecciones vaginales como la tricomoniasis se mostró en el 6\% entre los 21-35 años, que tiene similitud con los estudios realizados en el Hospital del Carmen en Manabí donde el 3\% se presentó entre los 19-30 años de edad (Aguirre, 2016), mientras que en embarazadas, que acuden al Centro de Salud de Catamayo se presentó un 3,33\% entre 23 29 años (Moreno, 2016). Resultados diferentes se observan en estudios realizados en pacientes atendidas en el Centro de Salud Santa Adriana de Perú donde un $27 \%$ presento infecciones vaginales por trichomona en mayores de 35 años (Tapia, 2020), mientras que en un estudio realizado en el Hospital Virgen de Remedios en Bolivia un mínimo porcentaje del $3,85 \%$ en la edad de 15 - 40 años (Cordero, 2018). En Esmeraldas la Tricomoniasis Vaginal en Embarazadas que acudieron al Hospital de Limones se mostró un 16,6\% entre los 18 - 38 años (Cabezas, 2020), estos estudios realizados nos revelan que la tricomoniasis se presenta con poca frecuencia en las mujeres embarazadas, más sin embargo se presenta en todas las edades.

En cuanto a la Candidiasis el $13 \%$ se presentó en la edad de 26 - 30 años, rango de edad que no coincide con estudios realizados con anterioridad, como el elaborado en pacientes gestantes de una Clínica de alta complejidad de la Ciudad de Medellín en Colombia donde existe un $41,4 \%$ en la edad de 23 años (Rojas, 2016), así mismo en un estudio realizado en el Centro de Salud Santa Adriana de Perú el $38 \%$ se mostró en mayores de 35 años (Tapia, 2020) y en Bolivia en el Hospital Virgen de Remedios del Municipio de Sopachuy el $65,38 \%$ en la edad de 15 - 40 años (Cordero, Flores, 2018), estos resultados muestran que la infección vaginal por Cándida en mujeres embarazadas se presenta con mayor frecuencia, seguida de la vaginosis bacteriana y la tricomoniasis.

Del $100 \%$ de las embarazadas que presentaron infecciones vaginales, el $4 \%$ presentó amenaza de aborto y el $96 \%$ no tuvo ninguna complicación, con un porcentaje más elevado en un estudio hecho en el Subcentro de Salud Unión de Ciudadelas de Quito siendo la única complicación amenaza de aborto con un 29,31\% (Vinueza, 2016), además se pudo revisar que en diferentes investigaciones existen otras complicaciones por infecciones vaginales como los realizados en embarazadas de Santa Cruz del Norte de Cuba donde el $11 \%$ de las pacientes en estudio presentó como complicación endometritis (Hernández, 2016), a diferencia de otro estudio realizado en el Subcentro De Salud De La Parroquia Cunchibamba" en Ambato en el cual el 17\% presentaron signos y síntomas de amenaza de parto pretérmino (Chacón, 2019), con un mínimo porcentaje en embarazadas de la maternidad Matilde Hidalgo De Procel del Guasmo Sur - Guayaquil donde el $4.4 \%$ presento parto pretérmino y el 6,6\% presentó ruptura prematura de membranas (Cruz, 2017), de tal modo se debe tomar en cuenta todas estas complicaciones en una paciente con infección vaginal, aunque el porcentaje de aparición de las mismas sea muy bajo.

En cuanto a la relación de infecciones vaginales con las complicaciones obstétricas que se producen del $100 \%$ de las pacientes en estudio el 48,07\% presentaron vaginosis bacteriana y el $42,30 \%$ candidiasis, presentando como complicaciones amenaza de aborto el $2,08 \%$ y $2,3 \%$ respectivamente. 
Se encontraron resultados diferentes puesto que en un estudio de tipo cuantitativo, observacional, analítico, de casos y controles retrospectivo realizado en el Área de maternidad del Hospital Vicente Corral Moscoso en Venezuela donde señaló que uno de los factores de riesgo para parto pretérmino es la vaginosis bacteriana con un 7,93\% (Cartillo, 2019), mientras que en Babahoyo la asociación de infecciones vaginales cervicales en el embarazo, se ha relacionado con trabajo de parto pretérmino en un 5\% - 10\% (Yépez, 2020) de tal forma se demuestra que las mujeres embarazadas con infecciones vaginales como vaginosis bacteriana, candidiasis y tricomoniasis están expuestas en un mínimo porcentaje a presentar alguna complicación obstétrica durante el curso de su gestación.

Es importante por ello trabajar constantemente en la prevención y promoción de las infecciones vaginales en el embarazo, evaluando a las pacientes en este estado de una manera adecuada en cada control prenatal. Además, es significativo el diagnóstico y tratamiento oportuno para evitar alguna complicación obstétrica que pueda afectar la salud materna o fetal.

\section{CONCLUSIONES}

Las infecciones vaginales más frecuentes que se presentaron en el embarazo es la vaginosis bacteriana en la edad de 21-30 años, seguido de la candidiasis en la edad de 26-30 años y la tricomoniasis en la edad de 21-35 años.

Dentro de las complicaciones obstétricas que se presentó por infecciones vaginales fue la amenaza de aborto en un mínimo porcentaje.

\section{AGRADECIMIENTOS}

Al finalizar con este trabajo de investigación, quiero expresar mi más sincero agradecimiento al personal del Centro Universitario de Motupe, al personal docente y a la Universidad Nacional de Loja por la colaboración en el proceso de esta investigación.

\section{CONTRIBUCIONES DE LOS AUTORES}

Conceptualización: MAS; metodología, VYG; investigación, VYG; recursos, MAS; curación de datos VYG; redacción, MAS; preparación de borrador original, MAS; redacción, VYG; revisión y edición, MAS y VYG; supervisión, MAS; adquisición de financiamiento VYG y MAS.

\section{FINANCIAMIENTO}

El financiamiento para el desarrollo de la investigación fue de procedencia propia, tanto del autor como coautor.

\section{REFERENCIAS}

Abdelaziz, Z. (2017). Vaginal infections among pregnant women at Omdurman Maternity Hospital in Khartoum. Sudan. J Infect Dev Ctries. Pag: 490.

Amaguaña, C. (2016). Influencia de las Infecciones Vaginales en la Amenaza de Parto Pretérmino en Pacientes Atendidas en el Subcentro de Salud de la Parroquia Cunchi- bamba.

Calderón, E y Arredondo, J. (2016). Complicaciones vaginales no inflamatorias durante la gestación. Infectología Perinatal. Primera edición. México, D.F. Editorial Trillas.

Calva Bereche, W. (2016). Complicaciones Obstétricas en Adolescentes Embarazadas Primigestas con Infección de Vías Urinarias Atendidas en el Servicio de Ginecoobstetricia del Hospital Isidro Ayora en la Ciudad de Loja. Repositorio de Universidad Nacional de Loja, Ecuador.

Carvajal A. (2016). Las cifras de embarazos en el país crecieron en niñas de 10 a 14 años. Recuperado de: http://www.elcomercio.com/tendencias/cifrasembarazosadolescentes-ecuadoreniplaplanfamiliaecuador.html

Chacón, S. (2019). Repositorio Universidad de Guayaquil: Prevalencia de infecciones vaginales en embarazadas y propuesta de un programa educativo, Subcentro de Salud Unión de Ciudadelas, Area de Salud No. 4 de Quito. Año 2012. Universidad de Guayaquil.

Charles, D y Eschenbach, D. (2013). Vaginosis durante la gestación: consecuencias y tratamiento. Infecciones Obstétricas y Perinatales. Primera edición. Madrid-España. Editorial Mosby/ Doyma - libros.

Cordero, M. y Flores, B. (2018). Vista de Frecuencia de Infecciones de Transmisión Sexual en Pacientes Gestantes, Bio Scientia.

Cunningham F, Gant N, y Leveno K. (2019). Infecciones de transmisión sexual. Mc Graw Hill, 25 ${ }^{\mathrm{a}}$ (Ed), (pp.12451247). México: McGraw-Hill Interamericana Editores, S.A. de C.V.

Guerra, M. A. D. J. (2016). Infección vaginal en gestantes y su incidencia en indicadores seleccionados del Programa Materno Infantil. Revista Cubana de Medicina General Integral.

Instituto Nacional de Estadísticas y Censos (2016). Características de las madres en el Ecuador.

Paavonen, J, y Brunham, R. (2019). Vaginosis bacteriana y vaginitis inflamatoria descamativa. Argentina. Artí 7 culos IntraMed.

Larry, J. (2019). Harrison: Principios de medicina interna. Vol. 1, 2 (20.a ed.). México. McGraw-Hill.

Loaiza, J., Romero, Y. y Albornoz, R. (2020). Universidad Ciencia y Tecnología. Perú.

Marcelo, A. (2016). Infecciones cérvico vaginales y embarazo. Revista Médica Clínica Las Condes.

Martínez, (2018). DSpace en Uniandes: Estrategia de prevención para disminuir la incidencia de vaginosis bacteriana en mujeres embarazadas atendidas en el hospital general Puyo en el periodo Junio 2017- Abril 2018. Repositorio 
Institucional UNIANDES.

Miranda A, Hernández LL y Romero C. (2016) Infección vaginal en gestantes y su incidencia en indicadores seleccionados del Programa Materno Infantil. Rev. Cubana Med Gen Integr Volumen 2, pag 291-300.

Moreira, R., Cunha, M., Borgues, B., Weyll, R., Teixeria, T., Silva, F., Rios, F. (2015). Prevalence and Risk Factors for Bacterial Vaginosis and other Vulvovaginitis in a population of sexually Active Adolescents from Salvador, Bahia, Brazil. Brasil.

MSP. (2014). Guía Práctica Clínica. Recuperado de: http://instituciones.msp.gob.ec/documentos/Guias/guias \%20 2014/GPC \%20Infeccion aginal $_{o}$ bstetrica.pdf

Nakano, F., De Barros, R., Esteves, S. (2015). Insights into the role of cervical mucus and vaginal $\mathrm{pH}$ in unexplained infertility. México

Pereira R, et al, (2015). Infección Vaginal en Gestantes. Revista Información Científica,

Pinheiro, P. (2016). Vaginosis Bacteriana Gardnerella Vaginalis.

Rojas, S. (2016). Infecciones vaginales en pacientes gestantes de una clínica de alta complejidad de MedellínColombia. Infecciones vaginales en pacientes gestantes de una clínica de alta complejidad de Medellín-Colombia, 16(No1), 32-42.

Tapia, F. (2020). Influencia de las características maternas sobre las infecciones vaginales en el Embarazo en Pacientes Atendidas en el Centro de Salud Santa Adriana. UNIVERSIDAD ANDINA NESTOR CACERES VELASQUEZ.

Topanda, F. (2016). Prevalencia de Vaginosis Bacteriana y su relación con los factores de riesgo asociados: El inicio temprano de relacione sexuales y el número de parejas sexuales en mujeres en edad fértil. Volumen 1. Pag 156.

Vega, María. (2016). Vaginitis. Revisión de guías clínicas. Servicio de Obstetricia y Ginecología.

Velázquez, G. (2016). Vulvovaginitis. SciELO Revista Científica de la UCSA. 